\title{
High-Harmonic Generation and Spin-Orbit Interaction of Light in a Relativistic Oscillating Window
}

\author{
Longqing Yi॰* \\ Department of Physics, Chalmers University of Technology, 41296 Gothenburg, Sweden
}

(Received 20 October 2020; revised 11 January 2021; accepted 8 March 2021; published 31 March 2021)

\begin{abstract}
When a high power laser beam irradiates a small aperture on a solid foil target, the strong laser field drives surface plasma oscillation at the periphery of this aperture, which acts as a "relativistic oscillating window." The diffracted light that travels though such an aperture contains high-harmonics of the fundamental laser frequency. When the driving laser beam is circularly polarized, the high-harmonic generation (HHG) process facilitates a conversion of the spin angular momentum of the fundamental light into the intrinsic orbital angular momentum of the harmonics. By means of theoretical modeling and fully 3D particle-in-cell simulations, it is shown the harmonic beams of order $n$ are optical vortices with topological charge $|l|=n-1$, and a power-law spectrum $I_{n} \propto n^{-3.5}$ is produced for sufficiently intense laser beams, where $I_{n}$ is the intensity of the $n$th harmonic. This work opens up a new realm of possibilities for producing intense extreme ultraviolet vortices, and diffraction-based HHG studies at relativistic intensities.
\end{abstract}

DOI: 10.1103/PhysRevLett.126.134801

Light carries angular momentum as spin and orbital components. The spin angular momentum (SAM) is associated with right or left circular polarization ( $\pm \hbar$ per photon), and the orbital angular momentum (OAM) is carried by light beams with helical phase fronts $\exp (i l \phi)$ ( $l \hbar$ per photon), also known as optical vortices, where $l$ is the topological charge and $\phi$ is the azimuthal angle [1]. The spin-orbit interaction of light refers to phenomena in which the spin affects the orbital degrees of freedom [2], such as spin-Hall effects [3,4]. Recently, interest in spin-orbit interaction has surged, as it not only gives physical insights into the behavior of polarized light at subwavelength scales, but also provides an important approach for producing optical vortices in the extreme ultraviolet (XUV) regime [5-9], that have a rich variety of applications in optical communication [10,11], biophotonics [12], and optical trapping [13].

Owing to the remarkable progresses in high-power lasers [14], such advanced light sources open up new possibilities in the relativistic regime $\left(>10^{18} \mathrm{~W} / \mathrm{cm}^{2}\right)$ of light-matter interactions [15-20], and can yield fundamental insights into the spin-orbit and orbit-orbit angular momentum interactions of relativistic light [21-24]. In particular, intense, ultrafast XUV vortices are of great interest for probing and manipulating the SAM and OAM of lightmatter interactions on the atomic scale. Most of the

Published by the American Physical Society under the terms of the Creative Commons Attribution 4.0 International license. Further distribution of this work must maintain attribution to the author(s) and the published article's title, journal citation, and DOI. Funded by Bibsam. proposed methods to produce such beams are based on high-harmonic generation (HHG) driven by relativistic vortex laser beams $[17,21,23]$, that are not widely available. Other techniques employ linearly polarized laser beams interacting with plasma holograms [18], or circularly polarized $(C P)$ laser pules irradiating a dented target $[25,26]$. However, these approaches rely on the relativistic oscillating mirror (ROM) mechanism [27-29] for producing harmonics, which is suppressed for $C P$ drivers at normal incidence $[29,30]$. Therefore it is challenging to generate intense circularly polarized vortex beams that are of particular interest for controlling chiral structures [31,32] and optical manipulation at relativistic intensities [33], due to the unique feature of constant ponderomotive force and donut-shaped intensity.

In this Letter, we introduce a new HHG mechanism based on light diffraction at relativistic intensities [34-36], which we call relativistic oscillating window (ROW). It allows for producing ultraintense circularly polarized XUV vortices with a high-power $C P$ laser beam. We show that when the laser pulse propagates through a small aperture on a thin foil, it drives chiral electron oscillation at the periphery, which results in spin-orbit interaction and HHG in the diffracted light.

We first demonstrate our scheme using 3D particle-in-cell (PIC) simulations with the code EPOCH [37]. The simulation setup and the main results are summarized in Fig. 1: a $C P$ laser beam propagates through a small aperture on a thin foil located at $x_{0}=4 \mu \mathrm{m}$. The laser field used in the simulation is $\mathbf{E}_{l}=\left(\mathbf{e}_{\mathbf{y}}+i \sigma \mathbf{e}_{\mathbf{z}}\right) E_{0} \sin ^{2}\left(\pi t / \tau_{0}\right) \exp \left(i k_{0} x-i \omega_{0} t\right)$, $0<t<\tau_{0}=54 \mathrm{fs}$, where $\mathbf{e}_{\mathbf{y}}\left(\mathbf{e}_{\mathbf{z}}\right)$ are the unit vectors 


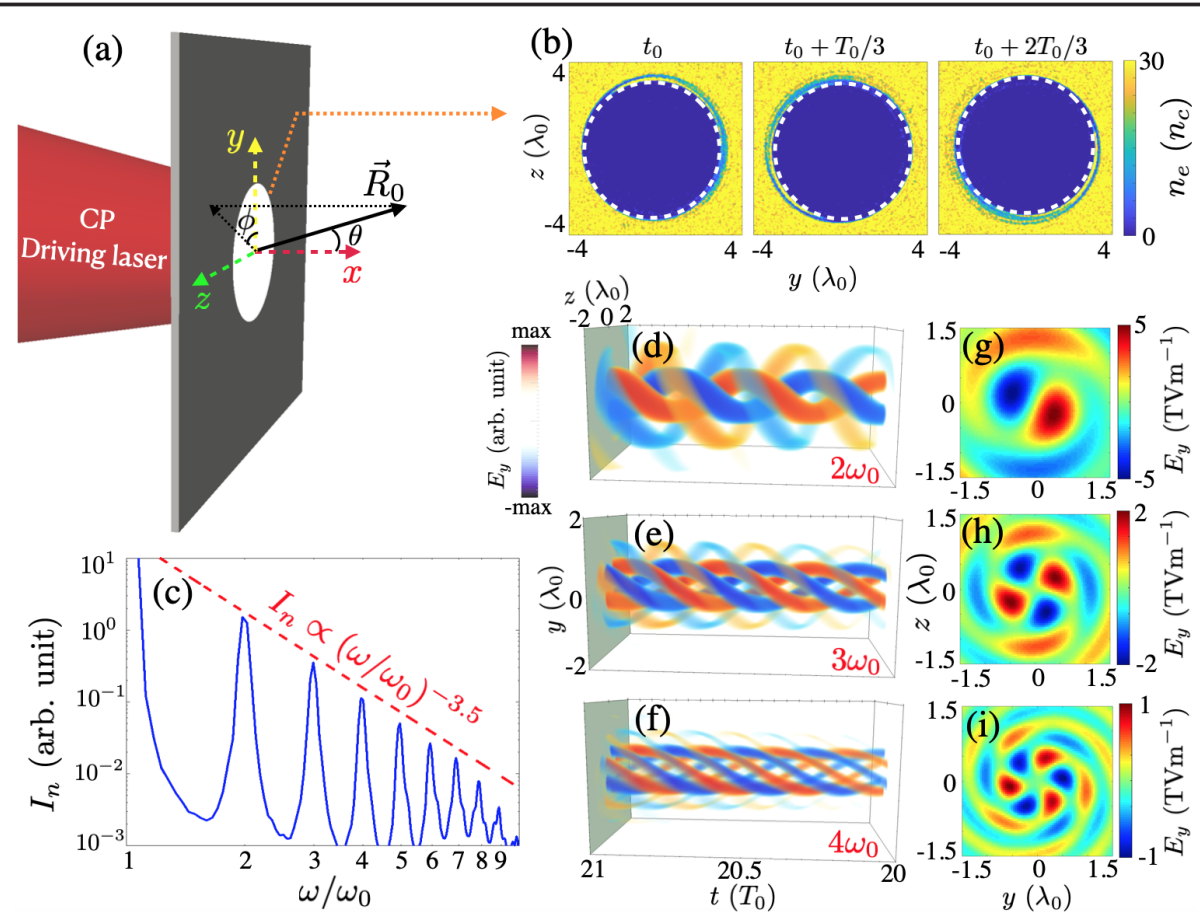

FIG. 1. (a) An intense $C P$ laser beam is focused on a foil with a small aperture, the laser field drives surface electron oscillation at the periphery, resulting in a dynamical electron density distribution (b). The three snapshots are separated temporally by a third of laser period $\left(T_{0}\right)$, from left to right, and the white dashed lines represent the boundary of a rigid oscillating window. (c) The spectrum of the diffracted light, the red dashed line represents a fitted power-law spectrum $I_{n} \propto n^{-3.5}$. (d)-(f) show the harmonic fields with frequency $2 \omega_{0}, 3 \omega_{0}$, and $4 \omega_{0}$, respectively. The field distributions in the 2D planes marked by dark green color in (d)-(f) are shown in (g)-(i), respectively.

in $\mathbf{y}(\mathbf{z})$ direction, $E_{0}$ is the laser amplitude, $k_{0}=2 \pi / \lambda_{0}$ the wave number, and $\lambda_{0}=1 \mu \mathrm{m}$ the wavelength. The laser polarization is controlled by $\sigma=+1$ and -1 for right- and left-handed circular polarization, respectively. The intensity of the laser beam is $I_{0} \approx 6.9 \times 10^{19} \mathrm{~W} / \mathrm{cm}^{2}$, corresponding to a normalized laser amplitude of $a_{0} \equiv e E_{0} / m_{\mathrm{e}} c \omega_{0}=5$, where $e, m_{\mathrm{e}}, c$ and $\omega_{0}$ denote the elementary charge, electron mass, vacuum light speed, and the laser frequency, respectively. The thin foil target [assumed plastic $(\mathrm{CH})$ ] is modeled by a preionized plasma with thickness $L_{f}=0.25 \mu \mathrm{m}$, and electron density $n_{0}=30 n_{c}$, where $n_{c}=m_{\mathrm{e}} \omega_{0}^{2} / 4 \pi e^{2} \approx 1.1 \times$ $10^{21} \mathrm{~cm}^{-3}$ is the critical density. The radius of the aperture is $r_{A}=4.0 \mu \mathrm{m}$, with a density gradient at the inner boundary $n(r)=n_{0} \exp \left[\left(r-r_{A}\right) / h\right]$ for $r<r_{A}$, where $h=0.2 \mu \mathrm{m}$ is the scale length. This yields an effective radius $r_{0}=3.3 \mu \mathrm{m}$, for which $n\left(r_{0}\right)=1 n_{c}$. The dimensions of the simulation box are $L_{x} \times L_{y} \times L_{z}=15 \mu \mathrm{m} \times 16 \mu \mathrm{m} \times 16 \mu \mathrm{m}$, sampled by $2400 \times 320 \times 320$ cells with fourteen macroparticles for electrons, two for $\mathrm{C}^{6+}$ and two for $\mathrm{H}^{+}$per cell. Mobile ions with real charge-to-mass ratio are used in the PIC simulations. A high-order particle shape function is applied to suppress numerical self-heating [37]. An open boundary condition is used in the $\pm x$ direction, while in the $\pm y$ and $\pm z$ directions, a periodic boundary condition is applied to launch a plane-wave laser pulse. This is justified as the size of the focal spot is assumed to be much larger than the aperture.
The intense laser field drives surface electron oscillations at the periphery, which modify the local plasma density as shown in Fig. 1(b). Since the region with electron density above $n_{c}$ is reflective to the laser pulse, the transparent area acts as a "relativistic oscillating window".

Figure 1(c) presents a typical spectrum of the diffracted light, which contains both even and odd orders of harmonics. It has a power-law shape that can be fitted by $I_{n} \propto n^{-3.5}$. The spectrum is obtained as the Fourier transform of the fields observed at a vertical plane $11 \mu \mathrm{m}$ away from the screen, within an opening angle of $\theta=30^{\circ}$.

Each harmonic with order $n$ is then selected by spectral filtering in the frequency range $[n-0.5, n+0.5] \omega_{0}$, shown in Figs. 1(d)-1(i). The spin-orbit interaction of light takes place, all harmonics are optical vortices with $|l|=n-1$.

Note that the underlying physics of the ROW, i.e., the chiral surface electron oscillation on the rim of the window, is a robust process for $C P$ light diffraction at relativistic intensities. The proposed scheme can work at both normal and oblique incidence, and a self-generated aperture can be relied on to overcome the alignment issue (see Supplemental Material [38]).

In this work, we restrict ourselves to the case of an intense $C P$ light diffracting through a predrilled aperture at close-to-normal incidence. In the following we consider the diffraction of a monochromatic plane wave 
$\mathbf{E}(x, y, z, t)=\mathbf{U}(x, y, z) \exp \left(-i \omega_{0} t\right)$ through an oscillating aperture, where $\mathbf{U}(x, y, z)$ satisfies Helmholtz equation $\left(\nabla^{2}+k_{0}^{2}\right) \mathbf{U}=0$. As the target is overdense, it is reasonable to assume the tangential components of the electric field vanish everywhere except in the aperture [29], where they can be approximated by that of the incoming laser fields. The diffracted field is given by the generalized Kirchhoff integral [39]:

$$
\begin{gathered}
\mathbf{E}_{\mathrm{diff}}(x, y, z, t)=\mathbf{U}(x, y, z) \exp \left(-i \omega_{0} t\right) \\
=\frac{1}{2 \pi} \nabla \times \int_{A}\left(\mathbf{e}_{\mathbf{n}} \times \mathbf{U}\right) \frac{\exp \left[i k_{0} R^{\prime}-i \omega_{0} t\right]}{R^{\prime}} d s^{\prime},
\end{gathered}
$$

where the integration is only over the aperture, $\mathbf{e}_{\mathbf{n}}$ is the unit vector normal to the screen. The distance between an observer at $(x, y, z)$ and elementary source $\left[d s^{\prime}\left(y^{\prime}, z^{\prime}\right)\right]$ is $R^{\prime}=\left|\mathbf{R}-d \mathbf{R}^{\prime}\right|$, measured at retarded time $t^{\prime}=t-R^{\prime} / c$. Here $\mathbf{R}$ is the initial distance, and $d \mathbf{R}^{\prime}\left(y^{\prime}, z^{\prime}, t^{\prime}\right)$ denotes the shift of $d s^{\prime}$ due to the strong laser field.

We now introduce the ROW model, it assumes that the shape of the aperture does not change (rigid window), such that each $d s^{\prime}$ is shifted by the same amount of displacement, $d \mathbf{R}^{\prime}\left(y^{\prime}, z^{\prime}, t^{\prime}\right)=d \mathbf{R}^{\prime}\left(t^{\prime}\right)$. This is valid for weakly relativistic drivers, where the surface electrons are simply shifted antiparallel to the driving laser field, resulting in a harmonic oscillation $d \mathbf{R}^{\prime}\left(t^{\prime}\right)=-\left(\mathbf{e}_{\mathbf{y}}+i \sigma \mathbf{e}_{\mathbf{z}}\right) \delta r_{0} \exp \left(-i \omega_{0} t^{\prime}\right)$, where $\delta r_{0}$ is the amplitude of the oscillation, for which values will be given below. To calculate the diffracted fields, one must solve for the retarded time $\left(t^{\prime}\right)$ numerically according to the motion of the source:

$R^{\prime}\left(t^{\prime}\right)=\left|\mathbf{R}+\left(\mathbf{e}_{\mathbf{y}}+i \sigma \mathbf{e}_{\mathbf{z}}\right) \delta r_{0} \exp \left[i k_{0} R^{\prime}\left(t^{\prime}\right)-i \omega_{0} t\right]\right|$.

However, to explain the spin-orbit interaction, it is sufficient to derive analytically the lowest order of diffracted fields, valid for $a_{0} \ll 1$, seen by a distant, paraxial observer, that satisfies $\left(R \gg r \gg r_{0}, \delta r_{0}\right)$. In this case we have $R^{\prime}\left(t^{\prime}\right) \approx R+\delta r_{0} \sin (\theta) \exp \left(i k_{0} R-i \omega_{0} t+i \sigma \phi\right)$, where $\theta=\arctan \left[r /\left(x-x_{0}\right)\right]$ and $\phi$ are defined Fig. 1(a). Substituting it into Eq. (1) and using the Jacobi-Anger identity [40] yields

$$
\begin{aligned}
\mathbf{E}_{\text {diff }} \approx & E_{0}\left(\mathbf{e}_{\mathbf{y}}+i \sigma \mathbf{e}_{\mathbf{z}}\right) \sum_{n=1}^{\infty} \frac{-i r_{0}}{2 \pi} \frac{J_{1}\left[n k_{0} r_{0} \sin (\theta)\right]}{\sin (\theta)} \\
& \times J_{n-1}(\epsilon) \frac{\exp \left[i n k_{0} R_{0}-i n \omega_{0} t+i(n-1) \sigma \phi\right]}{R_{0}},
\end{aligned}
$$

where $\epsilon=k_{0} \delta r_{0} \sin (\theta) \ll 1, R_{0}=\sqrt{\left(x-x_{0}\right)^{2}+y^{2}+z^{2}}$ is the distance measured from the initial center of the aperture, and $J_{n}$ are the Bessel functions of the first kind. The terms which are proportional to $\epsilon^{2}$ and smaller are neglected.

Equation (3) shows that HHG beams have helical phase fronts, with $l=(n-1) \sigma$ for the $n$th harmonic. It agrees well with the findings from PIC simulations. This relation guarantees the conservation of total angular momentum and energy: when $n$ photons at the fundamental frequency are transformed into one photon of $n$ th-order harmonic, their SAMs $(n \sigma \hbar)$ are converted into $(n-1) \sigma \hbar$ OAM plus $\sigma \hbar$ SAM.

In order to examine the ROW model at higher intensities, Eqs. (1) and (2) must be solved iteratively. Figure 2(a) presents a typical solution of Eq. (2) for the distance between the center of the window and an observer at $\theta=30^{\circ}$. It shows that due to the time it takes for the light to propagate, a harmonic oscillation of the source results in an anharmonic oscillation seen by the observer. This distortion due to retardation is the dominant mechanism to generate the high harmonics [28].

The HHG spectrum is then obtained by Fourier transforming the diffracted field calculated from Eq. (1). Figure 2(b) shows the spectra for weakly relativistic drivers. The harmonic intensities increase dramatically with laser $a_{0}$. In particular, the spectrum for small $a_{0}$ decays faster than exponentially with $n$, which agrees with Eq. (3) since $J_{n-1}(\epsilon) \sim(\epsilon / 2)^{n-1} /(n-1)$ !. As $a_{0}$ grows, the spectrum asymptotically converges to a power-law shape $I_{n} \propto n^{\alpha}$. This trend can be reproduced by our model as indicated by the open circles in Fig. 2(b).

Equation (2) suggests the amplitude of the oscillating velocity is $\delta r_{0} \omega_{0}$. Thus, substituting $\delta r_{0}<c / \omega_{0}$ into
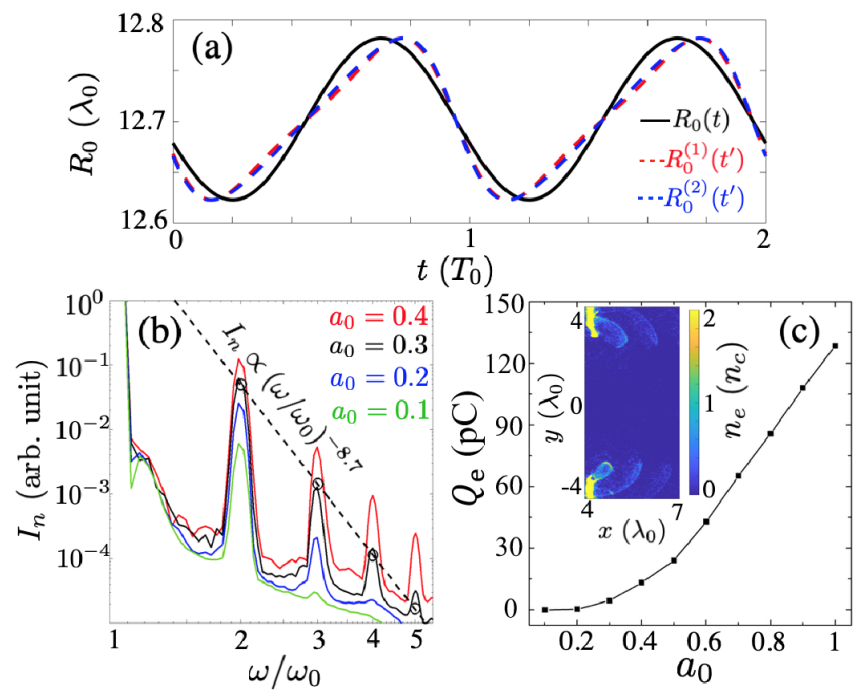

FIG. 2. (a) The distance between the ROW center and an observer. The harmonic oscillation of the window is presented by the black curve, and the motion seen by the observer, obtained by solving Eq. (2), are shown with the red and blue dashed lines, representing the results after one and two iterations, respectively. (b) HHG spectra (solid curves) for drivers with $a_{0}=0.1$ (green), 0.2 (blue), 0.3 (black), and 0.4 (red). The black open circles show the prediction of the ROW model with $\delta r_{0}=c / \omega_{0}$. (c) The total charge of the escaped electrons is plotted against the laser amplitude $a_{0}$, the inset shows a typical electron density distribution in $x-y$ plane, when SWB occurs. 
Eq. (1), one obtains the power-law exponent $\alpha<-8.7$, limited by causality. However, this is only true for $a_{0}<0.3$ according to Fig. 2(b), because at higher intensities, the electrons oscillating on the boundary of the aperture may gain enough energy to escape [41-43], as shown by the inset of Fig. 2(c). Therefore, the rim of the window can no longer be considered to be attached to these electrons, which significantly modifies the dynamics of the ROW.

This is due to surface wave breaking (SWB) [44]. To quantify when it should be taken into account, in Fig. 2(c) we plot the total charge of the escaping electrons as a function of the driving laser amplitude $a_{0}$. A surge of electron emission is observed for $a_{0}>0.3$, when the power-law exponents obtained from PIC simulations exceed -8.7 .

We now extend the ROW model to relativistic intensities $\left(a_{0}>1\right)$. Figure 3(a) shows a snapshot of typical plasma density distribution near the aperture when SWB occurs. The electrons can now travel far into the aperture when they oscillate inwards, the rim of the window on this side (red solid curve) follows the motion of the electrons for about half of one laser cycle, then it falls back to the original boundary as the electrons are emitted away and transparency is restored. On the other side (green solid curve), when the electrons travel towards the plasma bulk, the displacement remains small.

The diffracted field can then be calculated by separating the aperture into two parts, $A 1$ and $A 2$. As shown by Fig. 3(a), they are fractions of two rigid ROWs, which oscillate with different amplitudes $\delta r_{A 1}>\delta r_{A 2} \approx c / \omega_{0}$. The contributions from each part can then be obtained by integrating Eq. (1) over the area that satisfies $\mathbf{r} \cdot d \mathbf{R}^{\prime} \leq 0$ and $\mathbf{r} \cdot d \mathbf{R}^{\prime}>0$ for $A 1$ and $A 2$, respectively. In this way, the HHG spectra for $a_{0}>0.3$ can be reproduced from the model by adjusting the value of $\delta r_{A 1}$, as shown by Fig. 3(b). Setting $\delta r_{A 1}=0.25 \lambda_{0}$ and $0.4 \lambda_{0}$ recovers the HHG spectra from PIC simulations with $a_{0}=0.5$ and 1 (adjusted to the fifth harmonic), respectively. Notably, the power-law exponent depends very sensitively on the amplitude, therefore most of the harmonic signal comes from $A 1$ when SWB occurs.

The harmonic generation is enhanced dramatically by the SWB effect. In particular, the PIC simulations [Fig. 3(b)] suggest the power-law scaling is the same $\left(I_{n} \propto n^{-3.5}\right)$ for a sufficiently strong $\left(a_{0}>2\right) C P$ laser beam diffracting at close-to-normal incidence, which agrees well with the prediction from our model for $\delta r_{A 1}=0.5 \lambda_{0}$. This suggests the detailed electron dynamics at the periphery is not crucial for the HHG scaling; it is sufficient to consider a sinusoidal oscillation with an amplitude limited by causality. Because the electron layer can only travel inwards for less than half a laser cycle, the maximum displacement of the rim is $\sim c \times 0.5 T_{0}=0.5 \lambda_{0}$. In addition, both the model and simulations suggest this limit changes little with varying the aperture radius, two examples are given in Fig. 3(c).
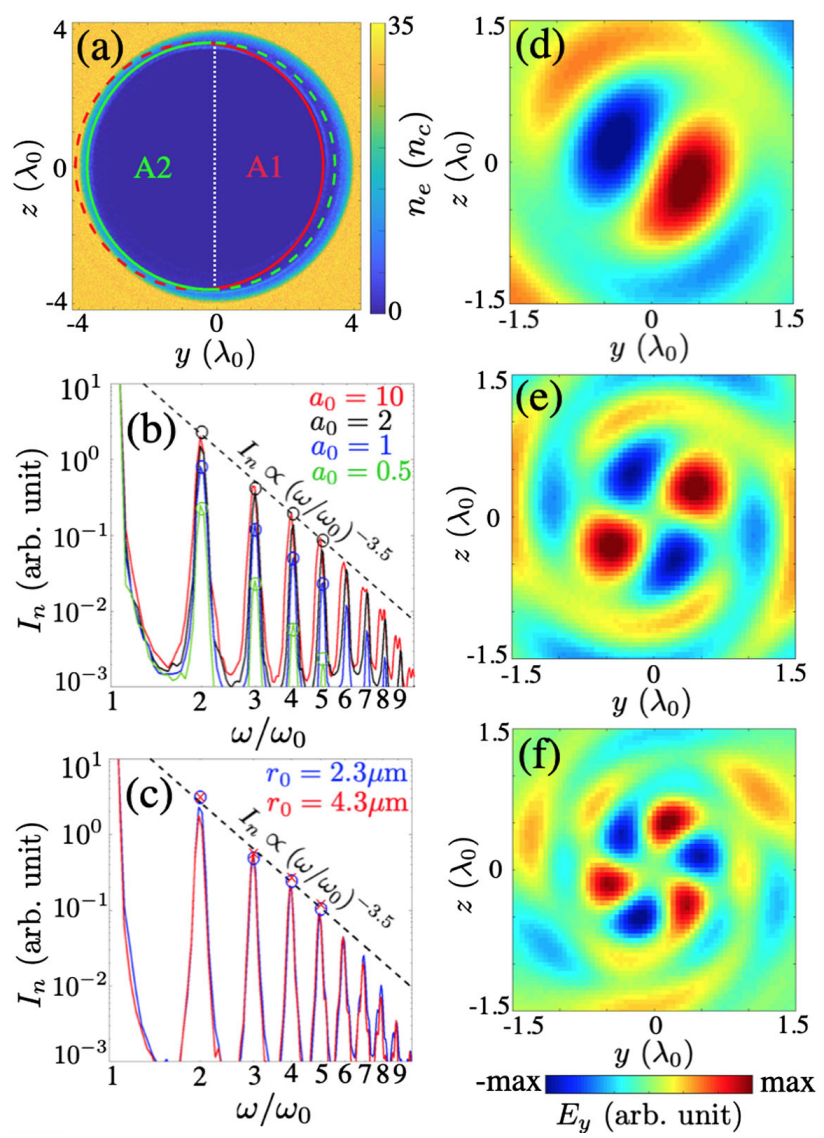

FIG. 3. (a) The electron density near the aperture when SWB occurs. The window (transparent area) is bounded by the solid curves (red and green). It can be separated into $A 1$ and $A 2$, which are fractions of two other rigid ROWs (red and green circles, consisting of both solid and dashed lines), with different amplitudes $\delta r_{A 1}>\delta r_{A 2} \approx c / \omega_{0}$. (b) HHG spectra from PIC simulations $\left(r_{0}=3.3 \lambda_{0}\right.$ is fixed) are shown by the solid curves for $a_{0}=0.5$ (green), 1 (blue), 2 (black), and 10 (red). The black open circles are the prediction of ROW model taking into account the SWB effect, with $\delta r_{A 1}=0.25 \lambda_{0}$ (green), $0.4 \lambda_{0}$ (blue), and $0.5 \lambda_{0}$ (black), respectively. (c) The HHG spectra with different radii $r_{0}=2.3 \mu \mathrm{m}$ and $4.3 \mu \mathrm{m}$, the PIC simulation data $\left(a_{0}=5\right.$ is fixed) are shown by the blue and red lines, while the results from the ROW model $\left(\delta r_{A 1}=0.5 \lambda_{0}\right)$ are presented by the blue open circles and red crosses, respectively. The $E_{y}$ field of the (d) second, (e) third, and (f) fourth harmonics obtained from the ROW model.

Note that the drop-off observed at eighth and ninth harmonics on the spectra is due to limited numerical resolution. We show with higher-resolution $2 \mathrm{D}$ simulations that the $I_{n} \propto n^{-3.5}$ scaling is retained to much higher harmonic numbers without significant drop-off (see Supplemental Material [38]).

Finally, the HHG fields can be obtained by filtering the diffracted fields calculated from Eq. (1) within a certain frequency range. Using the same parameters as in Fig. 1, and setting $\delta r_{A 1}=0.5 \lambda_{0}$, the corresponding second, third, and fourth harmonics are presented in Figs. 3(d)-3(f), 
respectively. Apparently the results confirm the relation $l=(n-1) \sigma$, and the harmonic fields agree very well with the PIC simulations shown in Figs. 1(g)-1(i).

In conclusion, we have demonstrated that high harmonics are generated when a high-power $C P$ laser pulse diffracts through a small aperture on a thin foil. In this process, the SAM of the driving laser beam is converted into OAM of the harmonics, giving rise to intense circularly polarized XUV vortices, with topological charge $l=(n-1) \sigma$ for the $n$th harmonic. By means of PIC simulation and semianalytical modeling, we show that the harmonic spectrum is $I_{n} \propto n^{-3.5}$, which does not depend very much on the driving laser intensity, provided that $a_{0}>2$. It would be interesting to examine this scaling at very large $a_{0}$, as for the ROM mechanism [45], this is left for future work.

The author acknowledges fruitful discussions with A. Pukhov, K. Hu, T. Fülöp, and I. Pusztai. This work is supported by the Olle Engqvist Foundation, the Knut and Alice Wallenberg Foundation and the European Research Council (ERC-2014-CoG Grant No. 647121). Simulations were performed on resources at Chalmers Centre for Computational Science and Engineering (C3SE) provided by the Swedish National Infrastructure for Computing (SNIC).

*lqyi@sjtu.edu.cn

[1] L. Allen, M. W. Beijersbergen, R. J. C. Spreeuw, and J. P. Woerdman, Phys. Rev. A 45, 8185 (1992).

[2] K. Y. Bliokh, F. J. Rodríguez-Frotuño, F. Nori, and A. V. Zayats, Nat. Photonics 9, 796 (2015).

[3] M. Onoda, S. Murakami, and N. Nagaosa, Phys. Rev. Lett. 93, 083901 (2004).

[4] O. Hosten and P. Kwiat, Science 319, 787 (2008).

[5] K. M. Dorney, L. Rego, N. J. Brooks, J. S. Román, C. T. Liao, J. L. Ellis, D. Zusin, C. Gentry, Q. L. Nguyen, J. M. Shaw, A. Picón, L. Plaja, H. C. Kapteyn, M. M. Murnane, and C. Hernández-García, Nat. Photonics 13, 123 (2019).

[6] M. Zürch, C. Kern, P. Hansinger, A. Dreischuh, and Ch. Spielmann, Nat. Phys. 8, 743 (2012).

[7] C. Hernández-García, A. Picón, J. San Román, and L. Plaja, Phys. Rev. Lett. 111, 083602 (2013).

[8] G. Gariepy, J. Leach, K. T. Kim, T. J. Hammond, E. Frumker, R. W. Boyd, and P. B. Corkum, Phys. Rev. Lett. 113, 153901 (2014).

[9] D. Gauthier, P. R. Ribic, G. Adhikary, A. Camper, C. Chappuis, R. Cucini, L. F. DiMauro, G. Dovillaire, F. Frassetto, R. Géneaux, P. Miotti, L. Poletto, B. Ressel, C. Spezzani, M. Stupar, T. Ruchon, and G. De Ninno, Nat. Commun. 8, 14971 (2017).

[10] J. Wang, J. Y. Yang, I. M. Fazal, N. Ahmed, Y. Yan, H. Huang, Y. X. Ren, Y. Yue, S. Dolinar, M. Tur, and A. E. Willner, Nat. Photonics 6, 488 (2012).

[11] G. Gibson, J. Courtial, M. J. Padgett, M. Vasnetsov, V. Pas'ko, S. M. Barnett, and S. Franke-Arnold, Opt. Express 12, 5448 (2004).
[12] K. I. Willig, S. O. Rizzoli, V. Westphal, R. Jahn, and S. W. Hell, Nature (London) 440, 935 (2006).

[13] A. T. O’Neil, I. MacVicar, L. Allen, and M. J. Padgett, Phys. Rev. Lett. 88, 053601 (2002).

[14] D. Strickland and G. Mourou, Opt. Commun. 55, 447 (1985).

[15] J. T. Mendonca, S. Ali, and B. Thidé, Phys. Plasmas 16, 112103 (2009).

[16] Y. Shi, B. F. Shen, L. G. Zhang, X. M. Zhang, W. P. Wang, and Z. Z. Xu, Phys. Rev. Lett. 112, 235001 (2014).

[17] J. Vieira, R. M. G. M. Trines, E. P. Alves, R. A. Fonseca, J. T. Mendonca, P. Norreys, and L. O. Silva, Nat. Commun. 7, 10371 (2016).

[18] A. Leblanc, A. Denoeud, L. Chopineau, G. Mennerat, Ph. Martin, and F. Quéré, Nat. Phys. 13, 440 (2017).

[19] J. Vieira, J. T. Mendonca, and F. Quéré, Phys. Rev. Lett. 121, 054801 (2018).

[20] W. P. Wang, C. Jiang, H. Dong, X. M. Lu, J. F. Li, R. J. Xu, Y. J. Sun, L. H. Yu, Z. Guo, X. Y. Liang, Y.X. Leng, R.X. Li, and Z.Z. Xu, Phys. Rev. Lett. 125, 034801 (2020).

[21] X. M. Zhang, B. F. Shen, Y. Shi, X. F. Wang, L. G. Zhang, W. P. Wang, J. C. Xu, L. Q. Yi, and Z. Z. Xu, Phys. Rev. Lett. 114, 173901 (2015).

[22] L. G. Zhang, B. F. Shen, X. M. Zhang, S. Huang, Y. Shi, C. Liu, W. P. Wang, J. C. Xu, Z. K. Pei, and Z.Z. Xu, Phys. Rev. Lett. 117, 113904 (2016).

[23] A. Denoeud, L. Chopineau, A. Leblanc, and F. Quéré, Phys. Rev. Lett. 118, 033902 (2017).

[24] Y. H. Tang, Z. Gong, J. Q. Yu, Y. R. Shou, and X. Q. Yan, Phys. Rev. E 100, 063203 (2019).

[25] J. W. Wang, M. Zepf, and S. G. Rykovanov, Nat. Commun. 10, 5554 (2019).

[26] S. S. Li, X. M. Zhang, W. F. Gong, Z. G. Bu, and B. F. Shen, New. J. Phys. 22, 013054 (2020).

[27] S. V. Bulanov, N. M. Naumova, and F. Pegoraro, Phys. Plasmas 1, 745 (1994).

[28] R. Lichters, J. Meyer-ter-Vehn, and A. Pukhov, Phys. Plasmas 3, 3425 (1996).

[29] T. Baeva, S. Gordienko, and A. Pukhov, Phys. Rev. E 74, 046404 (2006).

[30] Z. Chen and A. Pukhov, Nat. Commun. 7, 12515 (2016).

[31] K. Toyoda, K. Miyamoto, N. Aoki, R. Morita, and T. Omatsu, Nano Lett. 12, 3645 (2012).

[32] K. Toyoda, F. Takahashi, S. Takizawa, Y. Tokizane, K. Miyamoto, R. Morita, and T. Omatsu, Phys. Rev. Lett. 110, 143603 (2013).

[33] W. P. Wang, C. Jiang, B. F. Shen, F. Yuan, Z. M. Gan, H. Zhang, S. H. Zhai, and Z.Z. Xu, Phys. Rev. Lett. 122, 024801 (2019).

[34] B. Gonzalez-Izquierdo, R. J. Gray, M. King, R. J. Dance, R. Wilson, J. McCreadie, N. M. H. Butler, R. Capdessus, S. Hawkes, J.S. Green, M. Borghesi, D. Neely, and P. McKenna, Nat. Phys. 12, 505 (2016).

[35] B. Gonzalez-Izquierdo, M. King, R. J. Gray, R. Wilson, R. J. Dance, H. Powell, D. A. Maclellan, J. McCreadie, N. M. H. Butler, S. Hawkes, J.S. Green, C. D. Murphy, L. C. Stockhausen, D. C. Carroll, N. Booth, G. G. Scott, M. Borghesi, D. Neely, and P. McKenna, Nat. Commun. 7, 12891 (2016). 
[36] M. J. Duff, R. Wilson, M. King, B. Gonzalez-Izquierdo, A. Higginson, S. D. R. Williamson, Z. E. Davidson, R. Capdessus, N. Booth, S. Hawkes, D. Neely, R. J. Gray, and P. McKenna, Sci. Rep. 10, 105 (2020).

[37] T. D. Arber, K. Bennett, C. S. Brady, A. Lawrence-Douglas, M. G. Ramsay, N. J. Sircombe, P. Gillies, R. G. Evans, H. Schmitz, A. R. Bell, and C. P. Ridgers, Plasma Phys. Controlled Fusion 57, 113001 (2015).

[38] See Supplemental Material at http://link.aps.org/supplemental/ 10.1103/PhysRevLett.126.134801 for a discussion on the robustness of the ROW mechanism, and the cut-off of the harmonic spectra.

[39] J. D. Jackson, Classic Electrodynamics, 3rd ed. (Wiley, New York, 1999).
[40] A. A. M. Cuyt, V. Petersen, B. Verdonk, H. Waadeland, and W. B. Jones, Handbook of Continued Fractions For Special Functions (Springer, New York, 2008).

[41] N. Naumova, I. Sokolov, J. Nees, A. Maksimchuk, V. Yanovsky, and G. Mourou, Phys. Rev. Lett. 93, 195003 (2004).

[42] L. Q. Yi, A. Pukhov, P. Luu-Thanh, and B. F. Shen, Phys. Rev. Lett. 116, 115001 (2016).

[43] L. Q. Yi and T. Fülöp, Phys. Rev. Lett. 123, 094801 (2019).

[44] Y. Tian, J. Liu, W. Wang, C. Wang, A. Deng, C. Xia, W. Li, L. Cao, H. Lu, H. Zhang, Y. Hu, Y. Leng, R. Li, and Z. Xu, Phys. Rev. Lett. 109, 115002 (2012).

[45] M. R. Edwards and J. M. Mikhailova, Sci. Rep. 10, 5154 (2020). 